PLASTIC

BODIES 
EXPERIMENTAL FUTURES

Technological Lives, Scientific Arts, Anthropological Voices

A series edited by Michael M. J. Fischer and Joseph Dumit

\section{DUKE UNIVERSITY PRESS}

Durham and London

2016 


\title{
Plastic Bodies
}

\author{
Sex \\ Hormones \\ and Menstrual \\ Suppression \\ in Brazil
}

\author{
Emilia
}

Sanabria 
๑) 2016 Duke University Press

All rights reserved

Printed in the United States of America on acid-free paper @

Designed by Natalie F. Smith

Typeset in Quadraat Pro by Tseng Information Systems, Inc.

Library of Congress Cataloging-in-Publication Data Names: Sanabria, Emilia, [date] author.

Title: Plastic bodies : sex hormones and menstrual suppression in Brazil / Emilia Sanabria.

Other titles: Experimental futures.

Description: Durham : Duke University Press, 2016. | Series: Experimental futures: technological lives, scientific arts, anthropological voices | Includes bibliographical references and index. Identifiers: LCCN 2015039926| ISBN 9780822361428 (hardcover : alk. paper) | ISBN 9780822361619 (pbk. : alk. paper) | ISBN 9780822374190 (e-book)

Subjects: LCSH: Menstruation-Brazil-Salvador-Prevention. | Hormones, Sex. | Contraceptive drugs - Health aspects-BrazilSalvador. | Menstruation-Social aspects-Brazil-Salvador. | Menstrual regulation-Brazil-Salvador.

Classification: LCC QP263.S263 2016 | DDC 612.6/62098142-dc23

LC record available at http://lccn.loc.gov/2015039926

Cover art: David P. Wagner, Pill dispenser prototype for oral contraceptives, 1962 . Metal, plastic, wood, $1 / 4$ in. $\times 3^{1 / 8}$ in. Gift of David P. Wagner. Image courtesy of National Museum of American History, Kenneth E. Behring Center. 
TO MY MOTHER 
